\title{
Actividad lúdico formativa en personas mayores: campo de acción para los educadores y educadoras sociales ${ }^{11}$
}

Formative recreational activity in elder people: a scope for social educators

Dr. José Luis Rodríguez-Díez

Universidad Pablo de Olavide

jlrdiez@gmail.com

Dra. Victoria Pérez de Guzmán Puya

Universidad Pablo de Olavide

mvperpuy@upo.es

\section{Resumen}

En todo el mundo la proporción de mayores de 60 años está creciendo con más rapidez que ningún otro grupo etario, constituyendo en la actualidad un colectivo crucial dentro de las sociedades desarrolladas por su importante incidencia socioeconómica en las economías nacionales. La Encuesta de Presupuestos Familiares estimaba que el año 2006 el consumo de los adultos mayores en ocio ascendió a 8.812 millones de euros, lo que representaba el 0,9\% del PIB. Los/as adultos/as mayores han pasado de ser entendidos como una carga social pasiva, que recibe prestaciones y consume recursos, a ser considerados un colectivo que aportan y genera oportunidades y recursos a la sociedad. Las actividades en las que mayoritariamente participan son: Actividades deportivas $(26,6 \%)$; Formación $(12,2 \%)$ y Ocio turístico $(8,8 \%)$ y precisan profesionales del campo de la educación social formados en competencias específicas para poder trabajar con este colectivo.

\footnotetext{
${ }^{11}$ Recibido: 1/9/2015 Evaluado: 20/10/2015 Aceptado: 20/11/2015
} 


\title{
Palabras clave
}

Adultos mayores, economía, mercado laboral, actividades deportivas, formación, ocio turístico.

\begin{abstract}
Worldwide the proportion of people over 60 is growing faster than any other age group, becoming now a crucial group within developed societies for their important socioeconomic impact on national economies. The Household Budget Survey in the year 2006 estimated that consumption of the elderly in leisure amounted to 8.812 million euros, representing $0.9 \%$ of GDP. Older adults have gone from being understood as a passive social burden, which receives performances and consumes resources, to be considered a group that generates and provide opportunities and resources to society. The activities in which they are mostly involved are: sports activities (26.6\%), education (12.2\%) and leisure and tourism (8.8\%); so professionals in the field of social education trained in specific skills are required to work with this group.
\end{abstract}

\section{Keywords}

Elder adults, economy, labour market, sport activities, training, leisure tourism.

\section{Introducción}

La población mundial envejece, no sólo en el primer mundo sino en todo el planeta, de forma imparable y en un proceso sin precedentes en la historia de la humanidad, según el último informe de la División de Población de Naciones Unidas.

En todo el mundo la proporción de personas que tienen más de 60 años está creciendo con más rapidez que ningún otro grupo etario. Se prevé que en 2025 la cifra de personas con más de 60 años sea de 1.200 millones en el mundo y en 2050 serán una de cada tres. China va a pasar de un 12\% de mayores de 60 años a un $31 \%$ en solo 40 años; la India, de un $7 \%$ a un $20 \%$ y Brasil, de un $10 \%$ a un $29 \%$. (Cebeiro, 2010). El envejecimiento 
avanza muy rápido en los países en vías de desarrollo, sobre todo en las economías emergentes. En el siguiente gráfico (gráfico 1) exponemos la evolución mundial de habitantes mayores de 65 años por regiones (1955-2005), en millones.

Gráfico 1: Evolución mundial de habitantes mayores de 65 años por regiones (19552005).

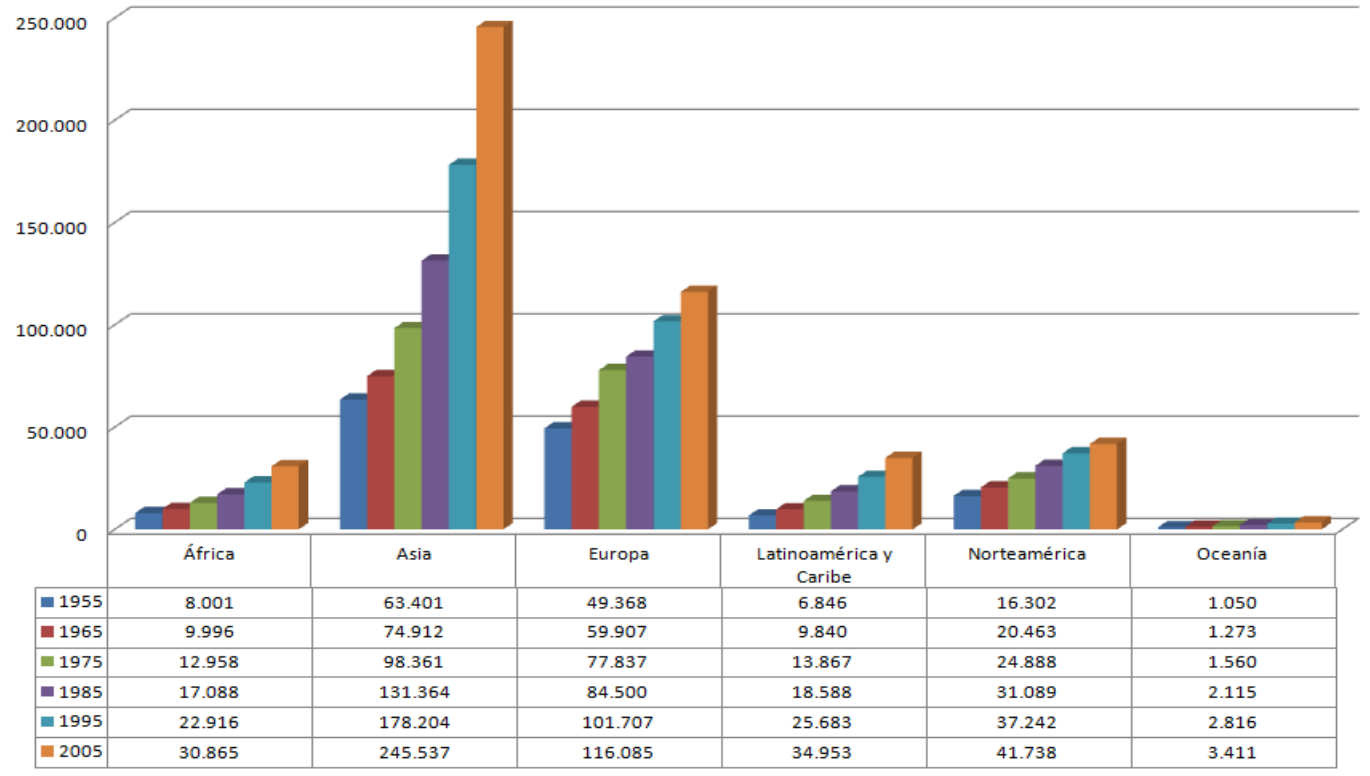

Fuente: Rodríguez-Díez, 2015 en base a ONU, 2009.

La humanidad se enfrenta al reto del envejecimiento de la población. Este envejecimiento puede considerarse un éxito de las políticas de salud pública y el desarrollo socioeconómico, pero también constituye un reto para la sociedad, que debe adaptarse a ello para mejorar al máximo la salud y la capacidad funcional de los adultos mayores, así como su participación social y su seguridad. Éste reto supone, además, la creación de innumerables puestos de trabajo directos e indirectos más allá de los relacionados directamente con la salud y/o los asistenciales debido a que las personas adultas mayores han pasado de ser entendidas como una carga social pasiva, que recibe prestaciones y consume recursos, a ser considerado un colectivo que aporta y genera oportunidades y recursos a la sociedad. 
Este sector constituye en la actualidad un colectivo crucial dentro de las sociedades desarrolladas por su importante incidencia socioeconómica dentro de las economías nacionales (Menéndez, 2008).

Es en la Segunda Asamblea Mundial sobre el Envejecimiento realizada en Madrid en el año 2002 donde se adoptaron, por primera vez, compromisos oficiales para responder a los desafíos que plantea el envejecimiento en todas sus dimensiones, ya que reconocieron el envejecimiento como un fenómeno mundial que implicaba transformaciones de toda índole en la sociedad. De esta Asamblea surge un documento que propone acciones concretas a desarrollar en tres áreas:

- Las personas de edad y el desarrollo

- El fomento de la salud y el bienestar en la vejez

- La creación de un entorno propicio y favorable

Tras esta Asamblea, se desarrolla la Conferencia Regional Intergubernamental sobre Envejecimiento (Santiago 2003), auspiciada por la Comisión Económica para América Latina y El Caribe (CEPAL), el Gobierno de Chile y los miembros del Grupo Interinstitucional sobre Envejecimiento (GIE) para analizar la implementación para América Latina y el Caribe del Plan de Acción Internacional de Madrid sobre el Envejecimiento. En este encuentro se plantean metas, objetivos y recomendaciones para la acción a favor de los adultos mayores en cada una de las áreas anteriormente expuestas.

Por tanto, aunque la perspectiva sobre el envejecimiento empieza a cambiar en las últimas décadas del siglo $\mathrm{XX}$, podemos considerar que es con el comienzo del siglo XXI cuando empieza a desarrollarse la idea del envejecimiento activo y saludable, entre otros motivos porque se pasa a tratar el envejecimiento desde un planteamiento basado en las necesidades, que contempla a las adultos mayores como objetivos pasivos, a otro basado en los derechos, que reconoce la igualdad de oportunidades, de trato y de participación en la vida comunitaria (OMS, 2002).

Actividades prioritarias que realizan las personas adultas mayores 
Según la Encuesta Mayores 2010 (IMSERSO, 2010) un 60\% de las personas adultas mayores inician nuevas actividades tras la jubilación. Numerosas investigaciones correlacionan la participación en actividades de tipo social, ya no sólo con la mejora en la calidad de vida de los y las mayores, sino con la mayor supervivencia (Sabin, 1993; Sugisawa, Liang \& Liu, 1994; Oxman, Freeman \& Manheimer, 1995; Lennartsson \& Silverstein, 2001 o Levy, 2003). Sin embargo, para que esta participación produzca el efecto anteriormente mencionado, las actividades deben causar placer y desarrollo, tanto en lo intelectual, como en lo social (Vega, Buz \& Bueno, 2002).

El verbo participar procede del latín participare. Tiene dos significados: tener parte y comunicar. Ambas acepciones tienen como origen común el término communis, que está estrechamente vinculado con comunidad. Por tanto, participación es una dimensión esencial de comunidad. (Vega, Buz \& Bueno, 2002). Según datos del IMSERSO (2010a) las actividades en las que mayoritariamente participan las personas adultas mayores son:

- Actividades deportivas, el 26,6\%

- Formación, el 12,2\%

- Ocio turístico, el 8,8\%

Es fundamental conocer estos tres campos de acción y/o intervención para poder ejercer en los mismos. En todos ellos ejercen su profesión educadores y educadoras sociales.

\section{Actividades deportivas}

La abundancia de programas de mantenimiento físico para personas adultas mayores, tanto de carácter público como privado, así como la interrelación entre el mayor nivel de bienestar, las mejores condiciones físicas, sociales y psicológicas y el aumento del tiempo libre, provocan que cada día un mayor número de estas personas realicen actividades deportivas.

Múltiples son los estudios epidemiológicos y longitudinales, en la población general, que han demostrado la importancia de la actividad física y deportiva en el mantenimiento de la salud y la calidad de vida (Paffenbarger et al., 1986; Paffenbarger 
\& Hide, 1988; Shephard, 1996; Alonso, 1997; Pieron, 2003; Mora et al., 2004, o Jiménez \& Montil, 2006 en Moscoso \& Moyano, 2009). La práctica deportiva regular promueve beneficios físicos, biológicos, psíquicos y sociales, y es importante tanto a nivel terapéutico como preventivo. Heredia (2006) considera que "el ejercicio físico practicado regularmente en la tercera edad contribuye a:

- Mejorar la capacidad para el auto cuidado.

- Favorecer la integración del esquema corporal.

- Propiciar bienestar general.

- Conservar más ágiles y atentos nuestros sentidos.

- Facilitar las relaciones inter-generacionales.

- Aumentar los contactos sociales y la participación social.

- Inducir cambios positivos en el estilo de vida de los adultos mayores.

- Incrementar la calidad del sueño.

- Disminuir la ansiedad, el insomnio y la depresión.

- Reforzar la actividad intelectual, gracias a la buena oxigenación cerebral.

- Contribuir en gran manera al equilibrio psico-afectivo.

- Prevenir caídas.

- Incrementar la capacidad aeróbica, la fuerza muscular y la flexibilidad.

- Disminuir el riesgo de enfermedad cardiovascular.

- Hacer más efectiva la contracción cardiaca.

- Frenar la atrofia muscular.

- Favorecer la movilidad articular.

- Evitar la descalcificación ósea.

- Aumentar la eliminación de colesterol, disminuyendo el riesgo de arteriosclerosis e hipertensión.

- Reducir el riesgo de formación de coágulos en los vasos y por tanto de trombosis y embolias.

- Aumentar la capacidad respiratoria y la oxigenación de la sangre.

- Evitar la obesidad.

- Mejorar la capacidad funcional de aquellos individuos que presentan un déficit en la realización de las $A V D$.

○ Incrementar la longevidad." (p 4)

Evidentemente, el ejercicio debe realizarse dentro de los parámetros de la salud y teniendo en cuenta las características personales de cada individuo. 


\section{Formación}

El interés por la vejez desde las Ciencias de la Educación no aparece hasta los años 60 del siglo pasado y se produce por un doble motivo: por la aparición y consolidación de programas educativos específicos para adultos mayores y por el comienzo de reflexiones teóricas que ofrecían soporte conceptual a esas prácticas educativas (Villar, 2001).

La educación de personas adultas mayores se ha concebido, desde la perspectiva del ciclo vital, en dos ámbitos de intervención:

- Educación compensatoria. Una vez la pérdida se ha producido, la educación podría actuar como una vía para remediarla. Los objetivos que persigue básicamente son (Villar, 2001; Sánchez, 2002):

- Dotar de las habilidades instrumentales básicas, como la lecto-escritura.

- Paliar las deficiencias asociadas al envejecimiento.

- Potenciar la actividad y relaciones sociales de la persona mayor.

- Educación como potenciadora del crecimiento. Concibe la educación centrada en el desarrollo, un medio para la adquisición de competencias nuevas.

Debemos distinguir entre los distintos tipos de formación dirigida a personas adultas mayores (Rodríguez-Díez, Amador, Pérez de Guzmán y Esteban, 2012):

- La Educación de Personas Adultas tiene como objetivos la adquisición, actualización y ampliación de conocimientos y aptitudes para mayores de 18 años.

- Las Aulas de la Tercera Edad, su objetivo es potenciar las capacidades lúdicas y creativas en los mayores, en torno a la cultura, así como posibilitar el desarrollo comunitario.

- Los Talleres Municipales para adultos mayores, su objetivo es ofrecer formación continua en distintos ámbitos de conocimientos y tareas ocupacionales. 
- Las Universidades Populares, sus objetivos están orientados a la formación de la persona, con contenidos muy variados, independientemente de la edad.

- Los Programas Universitarios para Mayores, sus objetivos son la formación, la investigación y la acción social.

La formación se ha convertido en una necesidad propiciada por diversos factores, entre los que están: exigencias democráticas, aumento del tiempo libre y ocio, incremento acelerado del conocimiento, las nuevas tecnologías de la información y la comunicación, democratización de la educación y la cultura y los grandes cambios sociales (Limón, 2009; Martínez-Mediano, Lord \& Riopérez, 2013). Ninguna generación en la historia de la Humanidad ha tenido que adaptarse a tantos cambios en tan poco tiempo (Rodríguez-Díez, Pérez de Guzmán, Amador y Vargas, 2012).

Los últimos datos oficiales que disponemos sobre el número de personas adultas mayores de 65 años que accedieron a formación formal o no formal en España, corresponden a la Encuesta sobre la participación de la población adulta en las actividades de aprendizaje (EADA, 2007) del INE, ya que en la EADA 2011 sólo se recogen datos de personas menores de 65 años.

En el año 2007, 3.730 .648 personas de entre 65 a 74 años participaron en actividades de educación formal y no formal (INE, 2007) y, según datos proporcionados por el Área de Participación Ciudadana del Excmo. Ayuntamiento de Sevilla, en el curso 2013-14 un total de 8.799 mayores de 65 años se matricularon en los distintos Talleres Municipales de Distrito y 4.000 más en el Programa Sociocultural para Mayores 2013-2014. Estos datos nos dan una idea de la importancia que ha tomado la formación en la vida de los adultos mayores actuales; así como la presencia de profesionales de la educación social que contribuyen al desarrollo de dichos programas.

\section{Ocio turístico}

Numerosas son las investigaciones que han comprobado que una mayor implicación en actividades de ocio por parte de los mayores se traduce en (García y Gómez, 2003): 
- Niveles de bienestar subjetivo más alto (Brown, Frankel y Fennell, 1991; Dupuis y Smale, 1995; Headey, Veenhoven y Wearing, 1991; Hersch, 1990; Lomranz, Bergman, Eyal y Shmotkin, 1988).

- Disminución del sentimiento de soledad (Lee e Ishii-Kuntz, 1987; Mullins y Mushel, 1992).

- Aumento del estado de ánimo (Lee e Ishii-Kuntz, 1987; Tinsley, Teaff, Colbs y Kaufman, 1987).

- Incremento en la capacidad de afrontamiento de los cambios producidos durante el envejecimiento (Kelly et al., 1986; Steinkamp y Kelly, 1987).

El turismo es ante todo una actividad, una práctica social (Álvarez Sousa, 1994), y varias son las variables que han propiciado el incremento exponencial que está teniendo el ocio turístico entre los mayores. El incremento de la esperanza de vida, los avances cualitativos en la calidad de vida de los mayores (Brewer, Poffley \& Pederson, 1995; Martín \& Guido, 1997), la capacidad de viajar en cualquier temporada y el crecimiento de su poder adquisitivo (Garau, 1998) han propiciado que para la industria turística este segmento de la población represente un mercado muy atractivo.

Por otro lado, las personas adultas mayores ya no entienden, solamente, el ocio turístico como la posibilidad de viajar, sino como una manera de obtener una experiencia vital satisfactoria que les proporcione bienestar y enriquecimiento personal (Marín, García \& Troyano, 2006).

Asimismo, desde la Administración Pública se potencia el turismo para mayores, ya que mantiene la actividad económica en este sector al favorecer la desestacionalización y generar recursos y empleo (Ortega, 1989). El programa de viajes del IMSERSO nació en 1985 y desde entonces más de doce millones de adultos mayores han viajado con él. Las plazas ofertadas fueron aumentando progresivamente desde las 16.000 plazas de la primera oferta en la temporada 1985/1986 hasta el 1.200.000 plazas de la temporada 2009/2010 (IMSERSO, 2010b), en la que la crisis también afectó a este programa y se 
empezaron a reducir el número de plazas, ofertándose 900.000 en la temporada $2013 / 2014$.

No entendemos estos recortes ya que estos programas son generadores de riqueza y empleo. Tanto es así que el Programa Vacaciones para Mayores en el curso 2009/2010 generó 16.000 puestos de trabajo directo y 102.000 puestos de trabajo indirectos (IMSERSO, 2010b), y el Programa de Termalismo Social, en el mismo periodo, generó 3.000 puestos de empleo directo y 21.000 de empleo indirecto (IMSERSO, 2010c). Cada Euro invertido en estos programas generó 1.54€ (IMSERSO, 2010b).

En 1998 la Fundación PriceWaterhouseCoopers afirmaba que el turismo para los mayores se está considerando y valorando como una necesidad social de primer orden considerando las estimaciones de crecimiento de este mercado y los efectos beneficiosos que produce.

Los programas de ocio-turismo desarrollados desde el IMSERSO son El Programa Vacaciones para Mayores y el Programa de Termalismo Social. El Programa Vacaciones para Mayores, plantea como objetivos:

- La mejora de la calidad de vida de los mayores.

- El mantenimiento del empleo del sector turístico.

- La potenciación de la actividad económica.

El IMSERSO se encarga de su organización y contrata la realización de los viajes a empresas de servicios. Pueden acceder al Programa los adultos mayores a partir de 65 años, los pensionistas de jubilación y otros pensionistas desde los 60 años. Al cónyuge no se le exige condición de edad ni pensión. Igualmente pueden beneficiarse del Programa las personas españolas residentes fuera de España. El usuario tiene derecho a los servicios de:

- Desplazamiento de ida y vuelta (excepto en los turnos sin transporte).

- Alojamiento con pensión completa.

- Habitación doble, o individual con suplemento.

- Menú de régimen, en caso necesario. 
- Asistencia sanitaria complementaria.

- Actividades de ocio y tiempo libre.

- Póliza colectiva de seguros. El Programa está muy bien valorado por los usuarios, el $96 \%$ de los participantes quiere repetir, y los aspectos más valorados son (IMSERSO, 2010b):

- El transporte

○ El alojamiento

- Las actividades de ocio y cultura

El Programa de Termalismo Social nació en 1989, y pretende entre sus objetivos:

- Proporcionar al colectivo de mayores tratamientos recuperadores en establecimientos termales especializados.

- Mejorar la calidad de vida de las adultos mayores.

- Favorecer la creación y el mantenimiento del empleo en la temporada baja del turismo.

Este programa tiene un fuerte impacto en la reducción del gasto farmacéutico y se ha constatado que una parte significativa de las personas que participan se sienten más "sanas" con la realización de los tratamientos termales (IMSERSO, 2010c).

La Encuesta de Presupuestos Familiares 2006 del Instituto Nacional de Estadística estimaba que el consumo de los adultos mayores en ocio ascendió a 8.812 millones de euros, lo que representaba el $0,9 \%$ del PIB de 2006. Siendo el 54\% en ocio, espectáculos y cultura y el $45 \%$ restante en gasto de hoteles, cafés y restaurantes.

\section{Trabajar con personas mayores: un campo profesional para los educadores $\mathbf{y}$ educadoras sociales}

Con esta aproximación a las actividades en las que participan la nueva generación de adultos mayores pretendemos concienciar, así como descubrir, las múltiples oportunidades laborales que se abren a la sociedad en general respecto de los adultos mayores. Lejos queda cuando la oferta laboral hacia este colectivo, como comentábamos anteriormente, se limitaba a las labores puramente asistenciales o de 
cuidado. Esta generación de adultos mayores en los países desarrollados se está convirtiendo, de forma directa o indirecta, en motor y revitalizador de la economía, y como tales precisan una nueva generación de trabajadores y trabajadoras formados en infinidad de ámbitos laborales y especializados en esta etapa vital. Desde monitores y monitoras de educación física, docentes, hasta los y las agentes de viaje, cualquier ámbito laboral necesita profesionales preparados para el trato con las personas adultas mayores.

Por ello, las oportunidades laborales que se ofrece en relación con este colectivo no pueden ser más amplias. Si bien, todo profesional debe partir de:

- Una práctica basada en la reflexión.

- Un pensamiento de dirigir sus acciones para crear y fomentar personas autónomas.

- Utilizar una metodología participativa.

- Entender el conflicto como necesario para el crecimiento.

Como indica Vargas Vergara (2011), es difícil transmitir algo que no se posee. Para formar en la reflexión con intenciones de ayudar al sujeto a conocerse a sí mismo para descubrir su proyecto profesional y vital, es necesario que la reflexión sea el punto de partida, medio y objetivo a alcanzar.

Para poder trabajar con personas adultas mayores, es necesario que los profesionales posean unas competencias básicas como son:

- Mediar e intervenir, asesorando, acompañando y promocionando a personas y grupos en situación de necesidad.

- Adquirir habilidades, destrezas y actitudes para la intervención socioeducativa.

- Afrontar los deberes y dilemas éticos con espíritu crítico, ante las nuevas demandas y formas de exclusión social que plantea la sociedad del conocimiento a la profesión del educador/a social.

- Poseer los conocimientos básicos sobre la educación de adultos y personas mayores. 
- Identificar y enmarcar los conocimientos dentro del paradigma de aprendizaje a lo largo de la vida.

- Saber diferenciar los enfoques y modelos epistemológicos que existen en torno a esta temática.

- Ser capaces de identificar los aspectos esenciales que diferencian la educación de personas adultas y mayores.

- Desarrollar la capacidad de escucha activa para el buen desarrollo de los procesos de comunicación dentro de la asignatura, con objeto de poder extrapolarlo para el buen desempeño profesional y demostrar respeto ante los demás.

- Desarrollar la capacidad creativa y crítica para realizar propuestas resolutivas ante posibles situaciones a resolver.

Trabajar en estos ámbitos profesionales supone constantes retos, por los cambios que se van produciendo constantemente. Si bien, es una puerta abierta a muchos profesionales del campo social; especialmente, a los educadores y las educadoras sociales.

\section{Referencia Bibliográfica}

Álvarez Sousa, A. (1994). El ocio turístico en las sociedades industriales avanzadas. Barcelona: Bosch

Brewer, K. P., Poffley, J. K. \& Pederson, E. B. (1995). Travel Interest among Special Seniors: Continuing Care Retirement Community Residents. Journal of Travel \& Tourism Marketing, 4 (2). 93-98.

Cebeiro, M. (19, 1,2010). El mundo entero envejece. El País, 45.

Garau, J. (1998). El turismo sénior, un segmento emergente: El caso de Baleares. En Primer Seminario de Marketing Turístico. Alicante, España, (AEDEMO). 271296.

García, M.A. \& Gómez, L. (2003) Efectos de los talleres de ocio sobre el bienestar subjetivo y la soledad en las personas mayores. Revista de Psicología Social, 18 (1), 35-47. 
Heredia, L. (2006). Ejercicio físico y deporte en los adultos mayores. GEROINFO. 1 (4). 1-10. Disponible en http://cort.as/aBLP (12-11-2015)

IMSERSO. (2010a). Encuesta a mayores 2010. Madrid: Ministerio de Sanidad y Política Social.

IMSERSO. (2010b). Vacaciones para personas mayores. Un Programa con grandes beneficios sociales. Madrid: Ministerio de Sanidad y Política Social.

IMSERSO. (2010c). Los Balnearios. La respuesta a su bienestar y salud. Madrid: Ministerio de Sanidad y Política Social.

INE. (2006). Encuesta de Presupuestos Familiares 2006. Madrid. INE.

INE. (2007). Encuesta sobre la Participación de la Población Adulta en las Actividades de Aprendizaje (EADA). Año 2007. Boletín informativo 3/2008.

Lennartsson, C. \& Silverstein, M. (2001). Does engagement with life enhance survival of elderly people in Sweden? The role of social and leisure activities. Journals of Gerontology: Social Sciences, 56, 335-342.

Levy, B. (2003). Mind Matters: Cognitive and Physical Effects of Aging SelfStereotypes. Journal of Gerontology: Psychological Sciences, 58(4). 203-211.

Limón, M.R. (2009). Estrategias educativas en la salud de las personas mayores. En R. Perea, (Dir.). Promoción y educación para la salud. Tendencias innovadoras (pp.169-206). Madrid: Díaz de Santos.

Marín, M., García, A. \& Troyano, Y. (2006). Modelo de ocio activo en las personas mayores. Revisión desde una perspectiva psicosocial. Revista Internacional de Ciencias Sociales y Humanidades, Sociotam. XVI (1.) 147-167.

Martín, L. \& Guido, M. (1997). Senior Citizens' Tourism: A Simple Approach to Determine their Future Travel Behavior. Revue de Tourisme, 3. 4-12. 
Martínez-Mediano, C., Lord, S. M., \& Losada, N. R. (2013). Programa de Desarrollo de Competencias para el Aprendizaje a lo Largo de la Vida para Estudiantes de Educación Superior. Pedagogía social. Revista interuniversitaria, (22), 137-151.

Menéndez, D. (2008). Economía y personas mayores. Madrid: IMSERSO.

Moscoso, D. \& Moyano, E (coord.) (2009). Deporte, salud y calidad de vida. Fundación La Caixa: Colección Estudios Sociales, nº 26

OMS. (2002). Envejecimiento activo: un marco político. Revista Española de Geriatría y Gerontología, 37(S2). 74-105

ONU. (2009). Informe sobre el estado de la población mundial. New York: Fondo de Población de las Naciones Unidas.

Ortega, E. (1989). Las vacaciones de los españoles de la Tercera Edad. Estudios Turísticos, 102 (6), 75-112.

Oxman, T.E., Freeman, D. H. \& Manheimer, E. D. (1995). Lack of social participation or religious strength and confort as risk factors for death after cardiac surgery in the elderly. Psychosomatic Medicine, 57 (1). 5-15.

PriceWaterhouseCoopers. (1998). Las Vacaciones para Mayores en su estado actual. Preferencias de los usuarios y efectos de una posible descentralización. Madrid: PriceWaterhouseCoopers.

Rodríguez-Díez, J.L. (2015). Repercusión de la formación en adultos mayores: el caso del Aula Abierta de mayores. Tesis Doctoral (no publicada). Sevilla: Universidad Pablo de Olavide.

Rodríguez-Díez, J.L., Amador, L.V., Pérez de Guzmán, V. \& Esteban, M. (2012). Nunca es tarde para aprender. Los Programas Universitarios para Mayores. Revista Decápolis 4, 60-63.

Rodríguez-Díez, J.L., Pérez de Guzmán, V., Amador, L.V. \& Vargas, M. (2012) Investigar en la Universidad de Mayores. Análisis de una experiencia. En Pérez 
Serrano, G. (Coord.) Envejecimiento Activo y Solidaridad Intergeneracional: Claves para un Envejecimiento Activo. (pp.1-16). Madrid, UNED.

Sabin, E. (1993). Social Relationships and Mortality among the Elderly. Journal of Applied Gerontology. 12 (1). 44-60.

Sánchez, M. (2002). Los programas universitarios para mayores: propósitos, razones y motivos. En J. Sáez (Ed.) Pedagogía Social y programas intergeneracionales: educación de personas mayores, (pp. 113-125). Málaga, Aljibe.

Sugisawa, H., Liang, J., \& Liu, X. (1994). Social networks, social support, and mortality among older people in Japan. Journal of Gerontology, 49 (1), 3-13.

Vargas Vergara, M. (2011). Espacio Europeo de Educación Superior. Análisis de una experiencia. Tesis Doctoral. Universidad Pablo de Olavide.

Vega, J.L., Buz, J. \& Bueno, B. (2002). Niveles de actividad y participación social en las personas mayores de 60 años. Revista Interuniversitaria del Profesorado, 45. $33-53$

Villar, F. (2001). ¿Tiene sentido la formación en la vejez? Las nuevas tecnologías como ejemplo paradigmático. Comunicación y Pedagogía, 173, 57-62. 